Pacific Journal of Mathematics

AN ELEMENTARY PROOF OF THE PRIME NUMBER 


\section{AN ELEMENTARY PROOF OF THE PRIME NUMBER THEOREM WITH REMAINDER TERM}

\section{ROBERT BREUSCH}

Introduction. In this paper, the prime number theorem in the form $\psi(x) \equiv \sum_{p^{m} \leqq x} \log p=x+o\left(x \cdot \log ^{-1 / 6+\varepsilon} x\right)$, for every $\varepsilon>0$, is established via a proof that in the well-known formula

$$
\rho(x) \equiv \sum_{p^{m} \leqq x} \frac{\log p}{p^{m}}=\log x+O(1) \equiv \log x+a_{x},
$$

$a_{x}=-A_{0}+o\left(\log ^{-1 / 6+\varepsilon} x\right)$. ( $A_{0}$ is Euler's constant. $)$

Throughout the paper, $p$ and $q$ stand for prime numbers, $k, m, n, t$, and others are positive integers, and $x, y$, and $z$ are positive real numbers.

Some well-known formulas, used in the proof, are

$$
\begin{aligned}
& \sum_{n \leqq x} \frac{\log ^{k} n}{n}=\frac{1}{k+1} \cdot \log ^{k+1} x+A_{k}+O\left(\frac{\log ^{k} x}{x}\right), \text { for } k=0,1, \ldots \\
& \sum_{y<n \leqq z} \frac{1}{n} \cdot \log ^{k}(n / y)=\frac{1}{k+1} \cdot \log ^{k+1}(z / y)+O\left(\frac{1}{y} \cdot \log ^{k}(z / y)\right),
\end{aligned}
$$

for $k=0,1, \cdots$

(3) $\sum_{n \leqq x} \log ^{k}(x / n)=O(x)$, for $k=1,2, \cdots$

(4) $\sum_{p^{m} \leqq x} \log p \cdot \log ^{k}\left(x / p^{m}\right)=O(x)$, for $k=0,1, \cdots$

( 5 ) $\sum_{n \leqq x} \mu(n) / n=O(1)(\mu(n)$ is Moebius' function.)

Two other formulas, used prominently, are

$$
\begin{aligned}
& \sigma(x) \equiv \sum_{p^{m} \leqq x} \frac{\log p}{p^{m}} \cdot \log \left(x / p^{m}\right)=\frac{1}{2} \cdot \log ^{2} x-A_{0} \cdot \log x+g_{x}\left(g_{x}=O(1)\right) \\
& \text { ( 7 ) } \tau(x) \equiv \sum_{p^{m} \leqq x} \frac{\log p}{p^{m}} \cdot \log ^{2}\left(x / p^{m}\right)=\frac{1}{3} \cdot \log ^{3} x-A_{0} \cdot \log ^{2} x \\
& +\left(2 \cdot A_{0}^{2}+4 \cdot A_{1}\right) \log x+O(1) .
\end{aligned}
$$

With the help of (1), (2), and (4), (6) can be proved easily :

$$
\begin{aligned}
\sigma(x) & =\sum_{p^{m} \leqq x} \frac{\log p}{p^{m}} \cdot\left(\sum_{n \leqq x / p^{m}} 1 / n-A_{0}+O\left(p^{m} / x\right)\right), \quad \text { or, with } k=n \cdot p^{m}, \\
\sigma(x) & =\sum_{k \leqq x} \frac{1}{k} \cdot \sum_{p^{m} l^{k}} \log p-A_{0} \cdot \log x+O(1) \\
& =\sum_{k \leqq x} \frac{\log k}{k}-A_{0} \log x+O(1)=\frac{1}{2} \cdot \log ^{2} x-A_{0} \log x+O(1) .
\end{aligned}
$$

Received December 31, 1958. 
Also, again with $k=n \cdot p^{m}$,

$$
\begin{aligned}
& \sum_{k \leqq x} \frac{\log k}{k} \cdot \log \frac{x}{k} \\
= & \sum_{k \leqq x} \frac{1}{k} \cdot \log \frac{x}{k} \cdot \sum_{p^{m} / k} \log p=\sum_{p^{m} \leqq x} \log p \cdot \sum_{n \leqq x / p^{m}} \frac{1}{n \cdot p^{m}} \cdot \log \left(\frac{x}{n \cdot p^{m}}\right) \\
= & \sum_{p^{m} \leqq x} \frac{\log p}{p^{m}} \cdot\left\{\log \left(\frac{x}{p^{m}}\right) \cdot \sum_{n \leqq x / p^{m}} \frac{1}{n}-\sum_{n \leqq x / p^{m}} \frac{\log n}{n}\right\} \\
= & \sum_{p^{m} \geqq x} \frac{\log p}{p^{m}} \cdot\left\{\log ^{2}\left(x / p^{m}\right)+A_{0} \log \left(x / p^{m}\right)-\frac{1}{2} \log ^{2}\left(x / p^{m}\right)-A_{1}\right\}+O(1) \\
= & \frac{1}{2} \cdot \tau(x)+A_{0} \cdot \sigma(x)-A_{1} \cdot \rho(x)+O(1) .
\end{aligned}
$$

(7) follows now by (1), (2), and (6).

The proof now proceeds in the following steps: in part I, certain asymptotic formulas for $a_{n}$ (see (1)) and $g_{n}$ (see (6)) are derived; they suggest that "on the average," $a_{n}$ is $-A_{0}$, and $g_{n}$ is $A_{0}^{2}+2 A_{1}$. In part II, formulas for $a_{n}$ and $g_{n}$ are derived which are of the type of Selberg's asymptotic formula for $\psi(x)$; part III contains the final proof.

\section{PART I}

First, the following five formulas will be derived; $K_{1}, K_{2}, \cdots$, are constants, independent of $x$.

$$
\begin{aligned}
& \sum_{n \leq x} \frac{1}{n} \cdot a_{n}=-A_{0} \log x+g_{x}+K_{2}+O\left(\frac{\log x}{x}\right) \\
& \sum_{n \leqq x} \frac{1}{n} \cdot a_{x / n}=-A_{0} \log x+K_{3}+O\left(\frac{\log x}{x}\right) \\
& \sum_{p \leqq x} \frac{\log p}{p^{m}} \cdot a_{p^{m}}=-A_{0} \log x+g_{x}+\frac{1}{2} a_{x}^{2}+K_{4}+O\left(\frac{\log x}{x}\right) \\
& \sum_{n \leqq x} \frac{1}{n} \cdot g_{n}=\left(A_{0}^{2}+2 \cdot A_{1}\right) \cdot \log x+O(1) \\
& \sum_{n \leqq x} \frac{1}{n} \cdot g_{x / n}=\left(A_{0}^{2}+2 \cdot A_{1}\right) \cdot \log x+K_{5}+O\left(\frac{\log ^{2} x}{x}\right) .
\end{aligned}
$$

Proofs.

$$
\begin{aligned}
\sigma(x) & =\sum_{n \leqq x} \log \frac{x}{n}(\rho(n)-\rho(n-1))=\sum_{n \leqq x} \rho(n) \cdot \log \frac{n+1}{n}+O\left(\frac{\log x}{x}\right) \\
& =\sum_{n \leqq x} \frac{\rho(n)}{n}+K_{1}+O\left(\frac{\log x}{x}\right) \\
& =\sum_{n \leqq x} \frac{\log n}{x}+\sum_{n \leqq x} \frac{1}{n} a_{n}+K_{1}+O\left(\frac{\log x}{x}\right) .
\end{aligned}
$$


(8) follows now from (6) and (2).

Also

$$
\begin{aligned}
\sum_{n \leqq x} \frac{1}{n} \cdot a_{x / n} & =\sum_{n \leqq x} \frac{1}{n} \cdot\left(\sum_{p^{m} \leqq x / n} \frac{\log p}{p^{m}}-\log \frac{x}{n}\right) \\
& =\sum_{k \leqq x} \frac{1}{k} \sum_{p^{m} / k} \log p-\sum_{n \leqq x} \frac{1}{n} \log \frac{x}{n} \quad\left(k=n \cdot p^{m}\right) \\
& =\sum_{k \leqq x} \frac{\log k}{k}-\sum_{n \leqq x} \frac{1}{n} \log \frac{x}{n} \text {. which proves (9) by (2). }
\end{aligned}
$$

And

$$
\begin{gathered}
\sum_{p^{m} \leqq x} \frac{\log p}{p^{m}} \cdot a_{p^{m}}=\sum_{p^{m} \leqq x} \frac{\log p}{p^{m}}\left(\sum_{t^{ \pm} \leqq p^{m}} \frac{\log q}{q^{t}}-\log \left(p^{m}\right)\right) \\
=\frac{1}{2}\left(\sum_{p^{m} \leqq x} \frac{\log p}{p^{m}}\right)^{2}+\frac{1}{2} \sum_{p^{m} \leqq x} \frac{\log ^{2} p}{p^{2 m}}-\log x \cdot \sum_{p^{m} \leqq x} \frac{\log p}{p^{m}}+\sum_{p^{m} \leqq x} \frac{\log p}{p^{m}} \log \frac{x}{p^{m}} .
\end{gathered}
$$

Thus, by (1), (2) and (6),

$$
\begin{aligned}
\sum_{p^{m} \leqq x} \frac{\log p}{p^{m}} \cdot a_{p^{m}}= & \frac{1}{2}\left(\log x+a_{x}\right)^{2}+K_{4}+O\left(\frac{\log x}{x}\right)-\log x \cdot\left(\log x+a_{x}\right) \\
& +\frac{1}{2} \log ^{2} x-A_{0} \log x+g_{x}, \quad \text { which proves }(10) .
\end{aligned}
$$

In the next proof, use is made of the easily established fact that

$$
\begin{aligned}
& \rho(n) \cdot \log \frac{n+1}{n}=\sigma(n+1)-\sigma(n) . \\
\tau(x)= & \sum_{n \leqq x} \log ^{2}\left(\frac{x}{n}\right)(\rho(n)-\rho(n-1)) \\
= & \sum_{n \leqq x} \rho(n)\left(\log ^{2}\left(\frac{x}{n}\right)-\log ^{2}\left(\frac{x}{n+1}\right)\right)+O(1) \\
= & \sum_{n \leqq x} \rho(n) \log \frac{n+1}{n} \cdot \log \frac{x^{2}}{n(n+1)}+O(1) \\
= & \sum_{n \leqq x}(\sigma(n+1)-\sigma(n)) \cdot \log \frac{x^{2}}{n(n+1)}+O(1) \\
= & \sum_{n \leqq x} \sigma(n) \cdot \log \frac{n+1}{n-1}+O(1)=\sum_{n \leqq x} \sigma(n) \cdot \frac{2}{n}+O(1) \\
= & \sum_{n \leqq x} \frac{\log ^{2} n}{n}-2 \cdot A_{0} \cdot \sum_{n \leqq x} \frac{\log n}{n}+2 \cdot \sum_{n \leqq x} \frac{1}{n} \cdot g_{n}+O(1) \quad(\text { by }(6)) .
\end{aligned}
$$

This proves (11), with the help of (2) and (7).

Finally

$$
\begin{array}{r}
\sum_{n \leq x} \frac{1}{n} \cdot g_{x / n}=\sum_{n \leq x} \frac{1}{n} \cdot\left(\sum_{p^{m} \leq x / n} \frac{\log p}{p^{m}} \cdot \log \frac{x}{n \cdot p^{m}}-\frac{1}{2} \log ^{2}\left(\frac{x}{n}\right)+A_{0} \log \frac{x}{n}\right), \\
\text { or, with } k=n \cdot p^{m},
\end{array}
$$




$$
\begin{aligned}
& \sum_{n \leqq x} \frac{1}{n} \cdot g_{x / n} \\
= & \sum_{k \leqq x} \frac{1}{k} \cdot \log \frac{x}{k} \cdot \sum_{p^{m} / k} \log p-\frac{1}{2} \cdot \sum_{n \leqq x} \frac{1}{n} \cdot \log ^{2}\left(\frac{x}{n}\right)+A_{0} \sum_{n \leqq x} \frac{1}{n} \cdot \log \frac{x}{n} \\
= & \sum_{k \leqq x} \frac{1}{k} \cdot \log \frac{x}{k} \cdot \log k-\frac{1}{2} \cdot \sum_{n \leqq x} \frac{1}{n} \cdot \log ^{2}\left(\frac{x}{n}\right)+A_{0} \sum_{n \leqq x} \frac{1}{n} \cdot \log \frac{x}{n} .
\end{aligned}
$$

(12) now follows by (2).

Formulas (8) through (12) suggest setting

$$
b_{x} \equiv a_{x}+A_{0}, \quad h_{x} \equiv g_{x}-\left(A_{0}^{2}+2 A_{1}\right) .
$$

In terms of $b_{x}$ and $h_{x}$, the five formulas read

$$
\begin{gathered}
\sum_{n \leqq x} \frac{1}{n} \cdot b_{n}=h_{x}+K_{6}+O\left(\frac{\log x}{x}\right) \\
\sum_{n \leqq x} \frac{1}{n} \cdot b_{x / n}=K_{7}+O\left(\frac{\log x}{x}\right) \\
\sum_{p^{m} \leqq x} \frac{\log p}{p^{m}} \cdot b_{p^{m}}=A_{0} \cdot\left(-A_{0}+b_{x}\right)+A_{0}^{2}+2 \cdot A_{1}+h_{x}+K_{4} \\
+\frac{1}{2} \cdot\left(-A_{0}+b_{x}\right)^{2}+O\left(\frac{\log x}{x}\right) \\
=h_{x}+\frac{1}{2} \cdot b_{x}^{2}+K_{8}+O\left(\frac{\log x}{x}\right) \\
\sum_{n \leqq x} \frac{1}{n} \cdot h_{n}=O(1) \\
\sum_{n \leqq x} \frac{1}{n} \cdot h_{x / n}=K_{9}+O\left(\frac{\log ^{2} x}{x}\right) .
\end{gathered}
$$

Next, it will be shown that

$$
\sum_{n \leq x} \frac{1}{n} \cdot b_{n}^{2}=\sum_{n \leq x} \frac{1}{n} \cdot b_{x / n}^{2}+O(1),
$$

and

$$
\sum_{n \leqq x} \frac{1}{n} \cdot b_{n} \cdot b_{x / n}=\sum_{p^{m} \leqq x} \frac{\log p}{p^{m}} \cdot h_{x / p^{m}}+O(1) .
$$

For a proof of (14), we know, by $\left(10^{\prime}\right)$, that

$$
\frac{1}{n} \cdot b_{n}^{2}=\frac{2}{n} \cdot \sum_{p^{m} \leqq n} \frac{\log p}{p^{m}} \cdot b_{p^{m}}-\frac{2}{n} \cdot h_{n}-\frac{2}{n} \cdot K_{8}+O\left(\frac{\log n}{n^{2}}\right),
$$

and

$$
\frac{1}{n} \cdot b_{x / n}^{2}=\frac{2}{n} \cdot \sum_{p^{m} \leqq x / n} \frac{\log p}{p^{m}} \cdot b_{p^{m}}-\frac{2}{n} \cdot h_{x / n}-\frac{2}{n} \cdot K_{8}+O\left(\frac{1}{x} \cdot \log \frac{x}{n}\right) .
$$


Thus, by (3), (11') and (12'),

$$
\begin{aligned}
\sum_{n \leqq x} \frac{1}{n} \cdot\left(b_{n}^{2}-b_{x / n}^{2}\right)=2 \cdot \sum_{n \leqq x} \frac{1}{n}\left(\sum_{p^{m} \leqq n} \frac{\log p}{p^{m}} \cdot b_{p^{m}}-\sum_{p^{m} \leqq x / n} \frac{\log p}{p^{m}} \cdot b_{p^{m}}\right)+O(1) \\
=2 \cdot \sum_{p^{m} \leqq x} \frac{\log p}{p^{m}} \cdot b_{p^{m}}\left(\sum_{p^{m} \leqq n \leqq x} \frac{1}{n}-\sum_{n \leqq x / p^{m}} \frac{1}{n}\right)+O(1) \\
=2 \cdot \sum_{p^{m} \leqq x} \frac{\log p}{p^{m}} \cdot b_{p^{m}} \cdot\left(\log \left(x / p^{m}\right)+O\left(1 / p^{m}\right)-\log \left(x / p^{m}\right)\right. \\
\left.-A_{0}-O\left(p^{m} / x\right)\right)+O(1)
\end{aligned}
$$

$=O(1)$, by $\left(10^{\prime}\right)$ and (4). This proves (14).

Also

$$
\begin{aligned}
\sum_{n \leqq x} \frac{1}{n} \cdot b_{n} \cdot b_{x / n} & =\sum_{n \leqq x} \frac{1}{n} \cdot b_{n} \cdot\left(\sum_{p^{m} \leqq x / n} \frac{\log p}{p^{m}}-\log \frac{x}{n}+A_{0}\right) \\
& =\sum_{n \leqq x} \frac{1}{n} \cdot b_{n} \cdot\left(\sum_{p^{m} \leqq x / n} \frac{\log p}{p^{m}}-\sum_{t \leqq x / n} \frac{1}{t}+2 \cdot A_{0}+O\left(\frac{n}{x}\right)\right) \\
& =\sum_{p^{m} \leqq x} \frac{\log p}{p^{m}} \sum_{n \leqq x / p^{m}} \frac{1}{n} b_{n}-\sum_{t \leqq x} \frac{1}{t} \sum_{n \leqq x / t} \frac{1}{n} b_{n}+O(1), \quad \text { by }\left(8^{\prime}\right) \\
& =\sum_{p^{m} \leqq x} \frac{\log p}{p^{m}} \cdot h_{x / p^{m}}+K_{6} \log x-\sum_{t \leqq x} \frac{1}{t} h_{x / t}-K_{6} \log x+O(1) \\
& =\sum_{p^{m} \leqq x} \frac{\log p}{p^{m}} \cdot h_{x / p^{m}}+O(1), \quad \text { by }\left(12^{\prime}\right) .
\end{aligned}
$$

From (14) and (15) it follows that

$$
\sum_{n \leqq x} \frac{1}{n} \cdot\left(b_{n} \pm b_{x / n}\right)^{2}=2 \cdot \sum_{n \leqq x} \frac{1}{n} \cdot b_{n}^{2} \pm 2 \cdot \sum_{p^{m} \leqq x} \frac{\log p}{p^{m}} \cdot h_{x / p^{m}}+O(1),
$$

and therefore

$$
\sum_{n \leqq x} \frac{1}{n} \cdot b_{n}^{2} \geqq\left|\sum_{p^{m} \leqq x} \frac{\log p}{p^{m}} \cdot h_{x / p^{m}}\right|+O(1)
$$

\section{PART II}

In the following, we shall employ the inversion formula

$$
G(x)=\sum_{n \leqq x} g\left(\frac{x}{n}\right) \quad \text { for all } x>0 \Rightarrow g(x)=\sum_{n \leqq x} \mu(n) \cdot G\left(\frac{x}{n}\right),
$$

as well as

$$
\sum_{n \leqq x} \frac{\mu(n)}{n} \log \frac{x}{n}=O(1) .
$$

For a proof of (17), we make use of the fact that $\sum_{n \leqq x} x / n=$ $x \cdot \log x+A_{0} x+O(1)$; thus, by the inversion formula, 


$$
x=\sum_{n \leqq x} \mu(n) \cdot \frac{x}{n} \log \frac{x}{n}+A_{0} \cdot \sum_{n \leqq x} \mu(n) \cdot \frac{x}{n}+O(x) .
$$

(17) follows now by (5).

If $f(x)$ is defined for $x>0$, then

$$
\begin{aligned}
& \sum_{n \leq x}\left\{\frac{x}{n} \cdot \log \frac{x}{n} \cdot f\left(\frac{x}{n}\right)+\frac{x}{n} \cdot \sum_{p^{m} \leqq x / n} \frac{\log p}{p^{m}} \cdot f\left(\frac{x}{n \cdot p^{m}}\right)\right\} \\
& =\sum_{n \leq x} \frac{x}{n} \cdot \log \frac{x}{n} \cdot f\left(\frac{x}{n}\right)+\sum_{k \leqq x} \frac{x}{k} \cdot f\left(\frac{x}{k}\right) \cdot \sum_{p^{m} / k} \log p \quad\left(k=n \cdot p^{m}\right) \\
& =\sum_{n \leq x} \frac{x}{n} \cdot \log \frac{x}{n} \cdot f\left(\frac{x}{n}\right)+\sum_{k \leqq x} \frac{x}{k} \cdot f\left(\frac{x}{k}\right) \cdot \log k=x \cdot \log x \cdot \sum_{n \leqq x} \frac{1}{n} \cdot f\left(\frac{x}{n}\right) .
\end{aligned}
$$

Thus, if we set

$$
F(x) \equiv x \cdot \log x \cdot \sum_{n \leqq x} \frac{1}{n} \cdot f\left(\frac{x}{n}\right),
$$

then, by the inversion formula,

$$
x \cdot \log x \cdot f(x)+x \cdot \sum_{p^{m} \leqq x} \frac{\log p}{p^{m}} \cdot f\left(x / p^{m}\right)=\sum_{n \leqq x} \mu(n) \cdot F\left(\frac{x}{n}\right) .^{1}
$$

In particular, if

$$
\sum_{n \leqq x} \frac{1}{n} \cdot f\left(\frac{x}{n}\right)=K+O\left(\frac{\log ^{k} x}{x}\right)
$$

then

$$
\sum_{n \leqq x} \mu(n) \cdot F\left(\frac{x}{n}\right)=K \cdot \sum_{n \leqq x} \mu(n) \cdot \frac{x}{n} \cdot \log \left(\frac{x}{n}\right)+O\left(\sum_{n \leqq x} \log ^{k+1}\left(\frac{x}{n}\right)\right)=O(x),
$$

by (17) and (3), and thus

$$
\begin{aligned}
& f(x) \cdot \log x+\sum_{p^{m} \leqq x} \frac{\log p}{p^{m}} \cdot f\left(x / p^{m}\right)=O(1), \\
& \text { if } \quad \sum_{n \leqq x} \frac{1}{n} \cdot f\left(\frac{x}{n}\right)=K+O\left(\frac{\log ^{k} x}{x}\right) .
\end{aligned}
$$

(Selberg's asymptotic formula for $\psi(x)$ corresponds to $f(x) \equiv \psi(x) / x-1$.) By $\left(9^{\prime}\right)$ and $\left(12^{\prime}\right), f(x) \equiv b_{x}$ and $f(x) \equiv h_{x}$ both satisfy the condition of (18), and thus

$$
\begin{aligned}
& b_{x} \cdot \log x+\sum_{p^{m} \leqq x} \frac{\log p}{p^{m}} \cdot b_{x / p^{m}}=O(1) \\
& h_{x} \cdot \log x+\sum_{p^{m} \leqq x} \frac{\log p}{p^{m}} \cdot h_{x / p^{m}}=O(1) .
\end{aligned}
$$

${ }^{1}$ Compare K. Iseki and T. Tatuzawa, "On Selberg's elementary proof of the prime number theorem." Proc. Jap. Acad. 27, 340-342 (1951). 
From (16) and (20) it follows that

$$
\sum_{n \leq x} \frac{1}{n} \cdot b_{n}^{2} \geqq\left|h_{x}\right| \cdot \log x+O(1) .
$$

If we add to (19)

$$
\left(\log x-A_{0}\right) \cdot \log x+\sum_{p^{m} \leqq x} \frac{\log p}{p^{m}} \cdot\left(\log \left(x / p^{m}\right)-A_{0}\right),
$$

which by (1) and (6) is equal to $3 / 2 \cdot \log ^{2} x-3 \cdot A_{0} \cdot \log x+O(1)$, we obtain

$$
\rho(x) \cdot \log x+\sum_{p^{m} \leqq x} \frac{\log p}{p^{m}} \cdot \rho\left(x / p^{m}\right)=\frac{3}{2} \cdot \log ^{2} x-3 \cdot A_{0} \cdot \log x+O(1) .
$$

If $0<c<1$, and $c \cdot x<y<x$, then it follows from the last equation that

$$
\begin{aligned}
\rho(x) \cdot \log x-\rho(y) \cdot \log y & \leqq \frac{3}{2} \cdot\left(\log ^{2} x-\log ^{2} y\right)+O(1) \\
& =\frac{3}{2} \cdot \log \frac{x}{y} \cdot(\log x+\log y)+O(1),
\end{aligned}
$$

$\log x \cdot(\rho(x)-\rho(y))+\log \frac{x}{y} \cdot \rho(y) \leqq \frac{3}{2} \cdot \log \frac{x}{y} \cdot(\log x+\log y)+O(1)$, or, since $\rho(y)=\log y+O(1)$,

$$
\begin{aligned}
\log x \cdot(\rho(x)-\rho(y)) & \leqq \log \frac{x}{y} \cdot\left(\frac{3}{2} \cdot \log x+\frac{1}{2} \cdot \log y\right)+O(1) \\
& <2 \cdot \log \frac{x}{y} \cdot \log x+O(1)
\end{aligned}
$$

Thus

$$
\rho(x)-\rho(y)<2 \cdot \log \frac{x}{y}+O\left(\frac{1}{\log x}\right),
$$

and, since $\rho(x)=\log x-A_{0}+b_{x}$, it follows that $b_{x}-b_{y}<\log x / y+$ $O(1 / \log x)$. Also obviously $b_{x}-b_{y} \geqq-\log x / y$, because $\rho(x)$ is nondecreasing. Thus we obtain

$$
\left|b_{x}-b_{y}\right| \leqq \log \frac{x}{y}+O\left(\frac{1}{\log x}\right) \text { if } c \cdot x<y<x, 0<c<1 .
$$

\section{PART III}

Let $B \geqq 1$ be an upper bound of $\left|b_{n}\right|$.

Since $b_{n}-b_{n-1}$ is either $-\log [n /(n-1)]$, or $\log p / n-\log [n /(n-1)]$, it cannot happen that $b_{n}=b_{n-1}=0$. 
Let the integers $r_{1}, r_{2}, \cdots, r_{t}, \cdots$ be the indices $n$ for which the $b_{n}$ change signs. Precisely :

$$
\left\{\begin{array}{l}
r_{1}=1 ; n=r_{t} \text { if } b_{n} \cdot b_{n+1} \leqq 0, \text { and } b_{n+1} \neq 0 ; \\
\text { if } r_{t}<v \leqq w<r_{t+1} \text { then } b_{v} \cdot b_{w}>0 ; \text { and } \\
\left|b_{r_{t}}\right|<\left(\log r_{t}\right) / r_{t} \text { for } t>1 .
\end{array}\right.
$$

Let $\left\{s_{k}\right\}$ be a sequence of integers, determined as follows: every $r_{t}$ is an $s_{k}$; if $\log \left(r_{t+1} / r_{t}\right)<7 \cdot B$, and $r_{t}=s_{k}$, then $r_{t+1}=s_{k+1}$; if $\log \left(r_{t+1} / r_{t}\right) \geqq 7 \cdot B$, enough integers $s_{k+v}$ are inserted between $r_{t}=s_{k}$ and $r_{t+1}=s_{k+m}$ such that $3 \cdot B \leqq \log \left(s_{k+v+1} / s_{k+v}\right)<7 \cdot B$, for $v=0,1, \cdots$, $m-1$. If there is a last $r_{t_{0}}=s_{k_{0}}$, a sequence $\left\{s_{k_{0}+v}\right\}$ is formed such that $3 \cdot B \leqq \log \left(s_{k_{0}+v+1} / s_{k+v}\right)<7 \cdot B$. Thus the $s_{k}$ form a sequence with the following properties:

$$
\left\{\begin{array}{l}
s_{1}=1 ; \log \left(s_{k+1} / s_{k}\right)<7 \cdot B ; \text { for } k>1, \text { either } \\
\log \left(s_{k+1} \mid s_{k}\right) \geqq 3 \cdot B \text { or }\left|b_{s_{k}}\right| \text { and }\left|b_{s_{k+1}}\right| \text { are both } \\
\operatorname{less} \text { than } \frac{\log s_{k}}{s_{k}} ; b_{v} \cdot b_{w}>0 \text { for } s_{k}<v \leqq w<s_{k+1} .
\end{array}\right.
$$

Assume now that $\alpha(0<\alpha<1 / 2)$ is such that

$$
\text { not } h_{x}=O\left(\log ^{-\alpha} x\right) \text {. }
$$

Then $\left|h_{x}\right| \cdot \log ^{\alpha} x$ is unbounded. Let $x$ be large, and such that $\left|h_{x}\right| \cdot \log ^{\alpha} x \geqq\left|h_{y}\right| \cdot \log ^{\alpha} y$ for all $y \leqq x$. Let $c$ and $d$ be positive integers such that

$$
s_{c-1}<\log x \leqq s_{c}, \text { and } s_{d} \leqq x<s_{d+1} .
$$

It will be shown that

$$
\frac{1}{2} \cdot(1-\alpha-o(1)) \cdot S(x) \leqq\left|h_{x}\right| \cdot \log x \leqq \frac{1}{3} \cdot(1+o(1)) \cdot S(x),
$$

where

$$
S(x) \equiv \sum_{k=c+1}^{a}\left|h_{s_{k}}-h_{s_{k-1}}\right| \cdot \log \left(s_{k} / s_{k-1}\right) .
$$

From this it will follow that $\alpha \geqq 1 / 3$.

Clearly

$$
\begin{aligned}
& \left|h_{x}\right| \cdot \log x=\left|h_{x}\right| \cdot \log ^{\alpha} x \cdot\left\{\log ^{1-\alpha} x-\log ^{1-\alpha} s_{d}+\sum_{k=2}^{a}\left(\log ^{1-\alpha} s_{k}-\log ^{1-\alpha} s_{k-1}\right)\right\} \\
& \quad \geqq \frac{1}{2} \cdot \sum_{k=c+1}^{a}\left(\left|h_{s_{k}}\right| \cdot \log ^{\alpha} s_{k}+\left|h_{s_{k-1}}\right| \cdot \log ^{\alpha} s_{k-1}\right) \cdot\left(\log ^{1-\alpha} s_{k}-\log ^{1-\alpha} s_{k-1}\right) \\
& \quad \geqq \frac{1}{2} \cdot \sum_{k=c+1}^{a}\left|h_{s_{k}}-h_{s_{k-1}}\right| \cdot \log ^{\alpha} s_{k-1} \cdot\left(\log ^{1-\alpha} s_{k}-\log ^{1-\alpha} s_{k-1}\right) .
\end{aligned}
$$


If $y<z$, it is easily shown by the mean value theorem that

$$
y^{\alpha} \cdot\left(z^{1-\alpha}-y^{1-\alpha}\right)>(1-\alpha) \cdot \frac{y}{z} \cdot(z-y)>\left(1-\alpha-\frac{z-y}{z}\right)(z-y) .
$$

With $y=\log s_{k-1}, z=\log s_{k}$, and from the fact that $s_{k}>\log x$, $\log \left(s_{k} / s_{k-1}\right)<7 \cdot B$, it follows by (27) that

$$
\left|h_{x}\right| \cdot \log x>\frac{1}{2} \cdot\left(1-\alpha-\frac{7 \cdot B}{\log \log x}\right) \cdot S(x) .
$$

For the next estimate, we need the following lemma.

Lemma. Let $v$ and $w$ be positive integers such that

(1) $\log \frac{w}{v}=O(1)$;

(2) $b_{n}>0$ for $v \leqq n \leqq w ;$

(3) $b_{v}<\frac{\log v}{v}$

Then

$$
\sum_{v \leqq n \leqq w} \frac{1}{n} \cdot b_{n}^{2} \leqq \frac{2}{3} \cdot \log \frac{w}{v} \cdot \sum_{v \leqq n \leqq w} \frac{1}{n} b_{n}+O\left(\frac{\log (w / v)}{\log v}\right) .
$$

Proof. If $b_{n} \leqq 1 / 3 \cdot \log w / v$ for every $n$ in $[v, w]$, the lemma is obviously correct. Otherwise, let $n_{1}$ be such that

$$
b_{n_{1}} \geqq \frac{1}{3} \cdot \log \frac{w}{v}, \quad b_{n}<\frac{1}{3} \cdot \log \frac{w}{v} \quad \text { for } \quad v \leqq n<n_{1} .
$$

If $\log \left(n_{1} / v\right)>1 / 3 \log (w / v)$, let $z\left(v \leqq z<n_{1}\right)$ be such that $\log \left(n_{1} / z\right)=$ $1 / 3 \log (w / v)$; otherwise, let $z=v$. Thus by (22), in every case, $\log \left(n_{1} / z\right)=1 / 3 \log (w / v)+O(1 / \log v)$. Clearly $b_{n}-2 / 3 \cdot \log w / v<0$ for $v \leqq n \leqq z$. Thus

$$
\begin{gathered}
T \equiv \sum_{v \leqq n \leqq w} \frac{1}{n} \cdot b_{n}^{2}-\frac{2}{3} \cdot \log \frac{w}{v} \cdot \sum_{v \leqq n \leqq w} \frac{1}{n} \cdot b_{n} \\
\leqq \sum_{z \leqq n \leqq w} \frac{1}{n} \cdot b_{n}^{2}-\frac{2}{3} \cdot \log \frac{w}{v} \cdot \sum_{z \leqq n \leqq w} \frac{1}{n} \cdot b_{n}, \\
T \leqq \sum_{z \leqq n \leqq w} \frac{1}{n} \cdot\left(b_{n}-\frac{1}{3} \cdot \log \frac{w}{v}\right)^{2}-\frac{1}{9} \cdot \log ^{2}(w / v) \cdot \log (w / z)+O\left(\frac{\log (w / v)}{v}\right) .
\end{gathered}
$$

By (22),

$$
\begin{aligned}
& \left|b_{n}-\frac{1}{3} \log (w / v)\right|=\left|b_{n}-b_{n_{1}}\right|+O\left(\frac{\log v}{v}\right) \\
\leqq & \left|\log \left(n_{1} / n\right)\right|+O\left(\frac{1}{\log v}\right)=\left|\log \left(n_{1} / z\right)-\log (n / z)\right|+O\left(\frac{1}{\log v}\right),
\end{aligned}
$$

and thus 


$$
\left|b_{n}-\frac{1}{3} \cdot \log \frac{w}{v}\right| \leqq\left|\log \frac{n}{z}-\frac{1}{3} \log \frac{w}{v}\right|+O\left(\frac{1}{\log v}\right) .
$$

Thus

$$
\begin{aligned}
T \leqq & \sum_{z \leqq n \leqq w} \frac{1}{n} \cdot\left(\log \frac{n}{z}-\frac{1}{3} \cdot \log \frac{w}{v}\right)^{2} \\
& -\frac{1}{9} \cdot \log ^{2}(w / v) \cdot \log (w / z)+O\left(\frac{\log (w / v)}{\log v}\right) \\
= & \sum_{z \leqq n \leqq w} \frac{1}{n} \cdot \log ^{2}(n / z)-\frac{2}{3} \cdot \log \frac{w}{v} \cdot \sum_{z \leqq n \leqq w} \frac{1}{n} \cdot \log \frac{n}{z}+O\left(\frac{\log (w / v)}{\log v}\right) \\
= & \frac{1}{3} \cdot \log ^{3}(w / z)-\frac{2}{3} \cdot \log ^{2}(w / v) \cdot \frac{1}{2} \cdot \log ^{2}(w / z)+O\left(\frac{\log (w / v)}{\log v}\right),
\end{aligned}
$$

by $\left(2^{\prime}\right)$, and thus $T \leqq O(\log (w / v) / \log v)$. This completes the proof of the lemma.

CoRollary 1. If condition (3) is replaced by $b_{w}<\log w / w$, the conclusion still holds; if $b_{n}<0$ in $v \leqq n \leqq w$, the conclusion holds if $b_{n}$ is replaced by $\left|b_{n}\right|$.

CoROllary 2. If instead of (3) it is known that $b_{v}<\log v / v$ and $b_{w}<\log w / w$ then

$$
\sum_{v \leqq n \leqq w} \frac{1}{n} \cdot b_{n}^{2} \leqq \frac{1}{3} \cdot \log \frac{w}{v} \cdot \sum_{v \leqq n \leqq w} \frac{1}{n} \cdot\left|b_{n}\right|+O\left(\frac{\log (w / v)}{\log v}\right) .
$$

For a proof, we split $[v, w]$ into two intervals by a division point at $(v \cdot w)^{1 / 2}$, and apply the lemma separately to each subinterval.

\section{Corollary 3.}

$$
\sum_{s_{k-1}<n \leqq s_{k}} \frac{1}{n} \cdot b_{n}^{2} \leqq \frac{1}{3} \cdot \log \left(s_{k} / s_{k-1}\right) \cdot \sum_{s_{k-1}<n \leqq s_{k}} \frac{1}{n} \cdot\left|b_{n}\right|+O\left(\frac{\log \left(s_{k} / s_{k-1}\right)}{\log s_{k}}\right) .
$$

Proof. If $\log \left(s_{k} / s_{k-1}\right)<3 \cdot B$, this follows from (24) and Corollary 2 ; if $\log \left(s_{k} / s_{k-1}\right) \geqq 3 B$, it is obvious, since $\left|b_{n}\right| \leqq B$.

By (26), $\sum_{n \leqq s_{c}} 1 / n \cdot b_{n}^{2}=O(\log \log x)$, and $\sum_{s_{d}<n \leqq x} 1 / n \cdot b_{n}^{2}=O(1)$; also

$$
\sum_{k=c+1}^{d} \frac{\log \left(s_{k} / s_{k-1}\right)}{\log s_{k}} \leqq \sum_{k=c+1}^{a} \log \left(\frac{\log s_{k}}{\log s_{k-1}}\right) \leqq \log \log x
$$

It follows from (29) that

$$
\sum_{n \leqq x} \frac{1}{n} \cdot b_{n}^{2} \leqq \frac{1}{3} \cdot \sum_{k=c+1}^{a} \log \left(s_{k} / s_{k-1}\right) \cdot \sum_{s_{k-1}<n \leqq s_{k}} \frac{1}{n} \cdot\left|b_{n}\right|+O(\log \log x) .
$$

By $\left(8^{\prime}\right) \sum_{s_{k-1}<n \leqq s_{k}} 1 / n \cdot\left|b_{n}\right|=\left|h_{s_{k}}-h_{s_{k-1}}\right|+O\left(\log s_{k} / s_{k}\right)$, and thus, by (21) and (27), 
$(30)$

$$
\left|h_{x}\right| \cdot \log x \leqq \frac{1}{3} \cdot S(x)+O(\log \log x) .
$$

It follows from (28) and (30) that

$$
\left[\frac{1}{3}-\frac{1}{2} \cdot\left(1-\alpha-\frac{7 \cdot B}{\log \log x}\right)\right] \cdot S(x) \geqq O(\log \log x),
$$

and since by (25) and (30) $S(x) \geqq K \cdot \log ^{1 / 2} x$, this implies that $\alpha \geqq 1 / 3$. Thus $h_{x}=o\left(\log ^{-1 / 3+\varepsilon} x\right)$, for every $\varepsilon>0$, and therefore, by $\left(8^{\prime}\right)$,

$$
\sum_{s_{k-1}<n \leqq s_{k}} \frac{1}{n} \cdot\left|b_{n}\right|=o\left(\log ^{-1 / 3+\varepsilon} s_{k}\right) \text {. }
$$

In order to find a bound for $\left|b_{x}\right|$, we consider now a particular interval $I_{k}=\left(s_{k-1}, s_{k}\right]$; let us assume that $b_{n}>0$ in $I_{k}$. Let $n_{2} \in I_{k}$ be such that $b_{n_{2}} \geqq b_{n}$ for every $n \in I_{k}$. Let $n_{1}\left(s_{k-1} \leqq n_{1}<n_{2}\right)$ be such that

$$
b_{n_{1}} \leqq \frac{1}{2} \cdot b_{n_{2}}<b_{n_{1}+1} .
$$

Then

$$
\sum_{n \in I_{k}} \frac{1}{n} \cdot b_{n}>\sum_{n=n_{1}+1}^{n_{2}} \frac{1}{n} \cdot b_{n}>\frac{1}{2} \cdot b_{n_{2}} \cdot \log \left(n_{2} / n_{1}\right)-O\left(1 / s_{k}\right) .
$$

But by (22),

$$
\log \left(n_{2} / n_{1}\right) \geqq b_{n_{2}}-b_{n_{1}}-O\left(\frac{1}{\log s_{k}}\right) \geqq \frac{1}{2} \cdot b_{n_{2}}-O\left(\frac{1}{\log s_{k}}\right) .
$$

Thus

$$
\sum_{n \in I_{k}} \frac{1}{n} \cdot b_{n}>\frac{1}{4} \cdot b_{n_{2}}^{2}-O\left(\frac{1}{\log s_{k}}\right) .
$$

It follows from (31) that $b_{n_{2}}^{2}=o\left(\log ^{-1 / 3+\varepsilon} n_{2}\right)$, and thus

$$
b_{x}=o\left(\log ^{-1 / 6+\varepsilon} x\right) \text {. }
$$

Finally,

$$
\begin{aligned}
\psi(x) & =\sum_{n \leqq x} n \cdot(\rho(n)-\rho(n-1))=[x] \cdot \rho([x])-\sum_{n \leqq x-1} \rho(n) \\
& =x \cdot\left(\log x-A_{0}+b_{x}\right)-\sum_{n \leqq x}\left(\log n-A_{0}+b_{n}\right)+O(\log x) \\
& =x \cdot \log x-A_{0} \cdot x+b_{x} \cdot x-x \cdot \log x+x+A_{0} \cdot x-\sum_{n \leqq x} b_{n}+O(\log x) \\
& =x+o\left(x \cdot \log ^{-1 / 6+\varepsilon} x\right)+o\left(\sum_{n \leqq x} \log ^{-1 / 6+\varepsilon} n\right), \quad \text { by (32). }
\end{aligned}
$$

The last sum is easily seen to be $o\left(x \cdot \log ^{-1 / 6+\varepsilon} x\right)$, and thus

$$
\psi(x)=x+o\left(x \cdot \log ^{-1 / 6+\varepsilon} x\right) .
$$





\section{PACIFIC JOURNAL OF MATHEMATICS}

\section{EDITORS}

David Gilbarg

Stanford University

Stanford, California

F. H. Brownell

University of Washington

Seattle 5, Washington
A. L. Whiteman

University of Southern Californıa Los Angeles 7, California

L. J. PaIge

University of California

Los Angeles 24, California

\section{ASSOCIATE EDITORS}
E. F. BECKENBACH
T. M. CHERRY
D. DERRY

E. HEWITT
A. HORN
L. NACHBIN
M. OHTSUKA

H. L. ROYDEN

M. M. SCHIFFER
E. SPANIER

E. G. STRAUS

F. WOLF

\section{SUPPORTING INSTITUTIONS}

\author{
UNIVERSITY OF BRITISH COLUMBIA \\ CALIFORNIA INSTITUTE OF TECHNOLOGY \\ UNIVERSITY OF CALIFORNIA \\ MONTANA STATE UNIVERSITY \\ UNIVERSITY OF NEVADA \\ NEW MEXICO STATE UNIVERSITY \\ OREGON STATE COLLEGE \\ UNIVERSITY OF OREGON \\ OSAKA UNIVERSITY \\ UNIVERSITY OF SOUTHERN CALIFORNIA
}

\author{
STANFORD UNIVERSITY \\ UNIVERSITY OF TOKYO \\ UNIVERSITY OF UTAH \\ WASHINGTON STATE COLLEGE \\ UNIVERSITY OF WASHINGTON \\ AMERICAN MATHEMATICAL SOCIETY \\ CALIFORNIA RESEARCH CORPORATION \\ HUGHES AIRCRAFT COMPANY \\ SPACE TECHNOLOGY LABORATORIES \\ NAVAL ORDNANCE TEST STATION
}

Mathematical papers intended for publication in the Pacific Journal of Mathematics should be typewritten (double spaced), and the author should keep a complete copy. Manuscripts may be sent to any one of the four editors. All other communications to the editors should be addressed to the managing editor, L. J. Paige at the University of California, Los Angeles 24, California.

50 reprints per author of each article are furnished free of charge; additional copies may be obtained at cost in multiples of 50 .

The Pacific Journal of Mathematics is published quarterly, in March, June, September, and December. The price per volume (4 numbers) is $\$ 12.00$; single issues, $\$ 3.50$. Back numbers are available. Special price to individual faculty members of supporting institutions and to individual members of the American Mathematical Society: $\$ 4.00$ per volume; single issues, $\$ 1.25$.

Subscriptions, orders for back numbers, and changes of address should be sent to Pacific Journal of Mathematics, 2120 Oxford Street, Berkeley 4, California.

Printed at Kokusai Bunken Insatsusha (International Academic Printing Co., Ltd.), No. 6, 2-chome, Fujimi-cho, Chiyoda-ku, Tokyo, Japan.

PUBLISHED BY PACIFIC JOURNAL OF MATHEMATICS, A NON-PROFIT CORPORATION

The Supporting Institutions listed above contribute to the cost of publication of this Journal, but they are not owners or publishers and have no responsibility for its content or policies. 


\section{Pacific Journal of Mathematics}

\section{Vol. 10, No. $2 \quad$ October, 1960}

Maynard G. Arsove, The Paley-Wiener theorem in metric linear spaces ........

Robert (Yisrael) John Aumann, Acceptable points in games of perfect

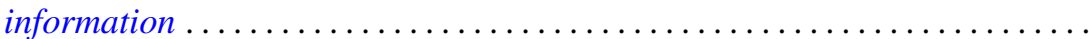

A. V. Balakrishnan, Fractional powers of closed operators and the semigroups generated by them ... . . . . . . . . . . . . . . . . . . . . . . . . . . . . 419

Dallas O. Banks, Bounds for the eigenvalues of some vibrating systems . . . . . 439

Billy Joe Boyer, On the summability of derived Fourier series . . . . . . . . . . . 475

Robert Breusch, An elementary proof of the prime number theorem with

remainder term ...................................

Edward David Callender, Jr., Hölder continuity of $n$-dimensional

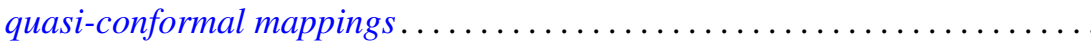

L. Carlitz, Note on Alder's polynomials ......................... 517

P. H. Doyle, III, Unions of cell pairs in $E^{3} \ldots \ldots \ldots \ldots \ldots \ldots \ldots \ldots \ldots \ldots \ldots \ldots . \ldots 21$

James Eells, Jr., A class of smooth bundles over a manifold . . . . . . . . . . . . 525

Shaul Foguel, Computations of the multiplicity function . . . . . . . . . . . . . . 539

James G. Glimm and Richard Vincent Kadison, Unitary operators in

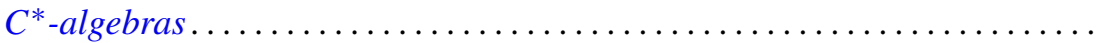

Hugh Gordon, Measure defined by abstract $L_{p}$ spaces . . . . . . . . . . . 557

Robert Clarke James, Separable conjugate spaces ....................

William Elliott Jenner, On non-associative algebras associated with bilinear forms

Harold H. Johnson, Terminating prolongation procedures

John W. Milnor and Edwin Spanier, Two remarks on fiber homotopy type .

Donald Alan Norton, A note on associativity . .

Ronald John Nunke, On the extensions of a torsion module.

Joseph J. Rotman, Mixed modules over valuations rings . . . . .

A. Sade, Théorie des systèmes demosiens de groupoï des . .

Wolfgang M. Schmidt, On normal numbers . .

661

Berthold Schweizer, Abe Sklar and Edward Oakley Thorp, The metrization of

statistical metric spaces

John P. Shanahan, On uniqueness questions for hyperbolic differential

equations

A. H. Stone, Sequences of coverings

Edward Oakley Thorp, Projections onto the subspace of compact operators

L. Bruce Treybig, Concerning certain locally peripherally separable spaces

Milo Wesley Weaver, On the commutativity of a correspondence and a

permutation

David Van Vranken Wend, On the zeros of solutions of some linear complex

differential equations. 